\title{
Midwives' Adherence to Preoperative Care Guidelines Prior to Emergency Caesarean Section at Bwaila Maternity Hospital in Malawi
}

\author{
Ruth Mwale1, Alfred Maluwa1 ${ }^{*}$, Address Malata1, Jon Odland ${ }^{2}$ \\ ${ }^{1}$ Kamuzu College of Nursing, University of Malawi, Lilongwe Campus, Lilongwe, Malawi \\ ${ }^{2}$ Norwegian Arctic University, Tromso, Norway \\ Email: aomaluwa@kcn.unima.mw
}

Received 1 September 2014; revised 29 September 2014; accepted 14 October 2014

Copyright (C) 2014 by authors and Scientific Research Publishing Inc.

This work is licensed under the Creative Commons Attribution International License (CC BY). http://creativecommons.org/licenses/by/4.0/

(c) (i) Open Access

\section{Abstract}

A study was conducted to describe midwives' adherence to preoperative care during emergency caesarian section at Bwaila Maternity Wing in 2012 in Malawi. The study utilized a descriptive prospective and retrospective design. A structured questionnaire was administered to all 28 midwives who were directly involved in the preparation of clients for emergency caesarean section. Clients charts were also reviewed using a standard checklist to determine adherence to preoperative care. Data were analyzed using SPSS version 16.0 and descriptive statistics in the form of frequencies and percentages were computed for the dataset. A midwife was supposed to score at least $80 \%$ on each component of preoperative care guidelines to be compliant to the stipulated pre-operation standards. The midwives scored above $80 \%$ on only four of the 14 preoperative care guidelines which were; bladder catheterization $(100 \%, n=14)$, obtaining informed consent $(92.9 \%, n=12)$, administration of IV $(96.4 \%, n=13)$ and administration of preoperative antibiotics $(82.1 \%, n=11)$. Midwives however scored less than $80 \%$ on preoperative procedures that dealt with vital signs $(28.6 \%, n=4)$ : BP check $(28.6 \%, n=4)$; Pulse rate check $(25 \%, n=3.5)$; respiration check $(25 \%, n=3.5)$ and temperature check $(25 \%, n=3.5)$. The midwives scored further below standard on blood specimen collection $(78.6 \%, n=11)$. Psychological support to clients was also below standard at $60.7 \%, n=8$. Other components of psychological support such as surgery information $(57.1 \%, n=8)$, allowing clients ask questions $(28.6 \%, n=4)$ and answering clients' questions $(25 \%, n=3.5)$ were also scored below standard. Overall the standard of preoperative care was below standard at the facility. Most of the midwives were new graduates, who had never received any in-service training on preoperative care. Therefore in service training it is recommended for the midwives to provide good quality of care.

\footnotetext{
Corresponding author.
} 


\section{Keywords}

\section{Emergency Caesarean Section, Preoperative Care Guidelines, Preoperative Vital Signs Check, Preoperative Psychological Support, Surgery Information, Pre-Operation Education}

\section{Introduction}

Clients undergoing emergency caesarean section (EmC/s) require good quality of care which prevents complications that are associated with EmC/s. Worldwide, 35.7\% of women suffer from major complications due to post caesarian section such as pelvic infection, sepsis, deep vein thrombosis, and other minor complications [1]. Major complications occur most frequently with emergency compared to elective caesarian sections. There is further evidence that EmC/s has a high risk of febrile morbidity, blood loss of more than 1000 ml, and an increased risk of a longer duration of postoperative stay of more than 7 days in hospitals [2].

In Africa, cesarean deliveries account for about 8.8\% of all births and are associated with maternal mortality ratios of 305 per 100,000 live births and still birth ratios of 36.6 per 1000 live births [3]. Caesarean deliveries account for 5\% - 21.8\% of all births in Sub-Saharan Africa and in Malawi they account for 3.7\% of all deliveries [4]. An estimated $94 \%$ of all the caesarian sections performed in Malawi are EmC/s, most of which are due to obstructed labor [5].

According to literature [6] [7], preoperative care should constitute: obtaining informed consent, administration of intravenous fluids, collection of blood specimens for hemoglobin grouping and cross matching, checking of vital signs, bladder catheterization and administration of preoperative antibiotics. Preoperative care also includes psychological care: teaching or educating the client on deep breathing and coughing exercises, turning exercises, leg exercises, and early ambulation.

In Malawi, the guidelines on preoperative care for emergency caesarean section give reference to obstructed labor, which is one of the indications of EmC/s [8]. These guidelines stipulate all the necessary preoperative care but they do not include obtaining informed consent, preoperative education and psychological care of the client [8].

Generally it has been observed that the care provided to clients undergoing EmC/s varies according to who has prepared the client before operation. Most of these clients are from rural areas, where care is of poor quality before arriving at the referral hospital. Clients therefore suffer from severe dehydration, anemia, and/or sepsis thus putting them at increased risk of morbidity and mortality. Nurses and midwives have a major role to play in the preoperative care of clients to reduce morbidity and mortality in the postoperative period [9]. Unpublished data at the facility indicate that of the 1433 mothers who had EmC/s in 2008, a total of 34 (2.4\%) developed puerperal sepsis, 23 (1.6\%) had gaping wounds, and 25 (1.7\%) were anemic postoperatively. Although many studies have been done on caesarean section in general, little is known on the quality of preoperative care that is rendered to mothers undergoing EmC/s in Malawi. The purpose of this study was therefore to describe the midwives' adherence to preoperative guidelines during the preparation of clients prior to EmC/s at Bwaila Maternity Unit in Lilongwe, Malawi.

\section{Methodology}

\subsection{Design}

The study design was descriptive prospective and retrospective and employed quantitative data collection and analysis method.

\subsection{Setting}

The study was conducted at Bwaila Maternity Unit in Lilongwe District of Central Malawi from September to October, 2012. Bwaila maternity unit serves a population of 2,203,911. Out of this 506,900 were women of child bearing age [10]. In 2008, a total of 12,000 deliveries were conducted at the hospital, out of which 1524 were caesarian sections. There were 28 midwives at the maternity out of which 19 were working in the labor ward and 9 in the antenatal ward. These midwives were responsible for providing preoperative care for EmC/s. 


\subsection{Sample}

The study targeted all available and consenting midwives at the facility during the time of study. All the 28 midwives that were available at the maternity and antenatal wards consented and participated in the study.

\subsection{Inclusion and Exclusion Criteria}

The study included all consenting midwives that were working at the antenatal and labor wards of Bwaila Maternity Unit, regardless of cadre but rendered preoperative care for EmC/s. The study did not recruit midwives that were working at Bwaila on part time basis or those working in other wards other than antenatal, labor and delivery.

\subsection{Data Collection}

A structured questionnaire was used to collect data from the midwives. The instrument consisted of three sections; the first section collected demographic data of the midwives. The second part collected data regarding participants' awareness on the components of preoperative care for EmC/s as stipulated in the RH guidelines [8]. Also included was data on obtaining informed consent, psychological care and client education, which were missing in the RH guidelines. The third part collected data on midwives' source of knowledge for the preoperative care they rendered and whether guidelines for preoperative care for $\mathrm{EmC} / \mathrm{s}$ were available in their wards. The interviews took approximately 30 to 45 minutes. Data for the documentation was obtained from review of 87 clients' charts using a standard recommended RH checklist [11].

\subsection{Data Analysis}

Data were analyzed using SPSS Version 16.0. Descriptive statistics were computed for the dataset and the results are presented as percentages across each component of preoperative guidelines. The midwives' mean percentage scores for each component of care were compared to the stipulated reproductive health guidelines. According to the reproductive health guidelines [8], midwives are viewed to be compliant with the standards if their mean scores are at least $80 \%$ on each of the stipulated standards in the guidelines.

\subsection{Ethical Consideration}

The research protocol was approved by the College of Medicine Research and Ethics Committee (COMREC) the internal ethical review board for Kamuzu College of Nursing. Permission was obtained from the District Health Officer for Lilongwe to conduct research at Bwaila Maternity Unit. Informed consent was obtained from each participant before they participated in the study. Other ethical issues such as maintaining confidentiality and anonymity were strictly observed and participants were given the freedom to withdraw from the study anytime if they wished to do so.

\section{Results}

\subsection{Midwives' Demographic Characteristics}

The midwives' ages ranged from 25 to over 50 years, with a mean of 37.5 years but the majority (39.3\%) was aged less 30 years. Regarding their education, most of the participants $(89.3 \%, n=25)$ had Malawi School Certificate of Education (MSCE). The participants comprised different cadres with most of them being the Nurse Midwife Technicians $(39.3 \%, \mathrm{n}=11)$. Enrolled Nurse Midwives comprised $(35.7 \%, \mathrm{n}=10)$. There were few registered nurse midwives $(21.4 \%, \mathrm{n}=6)$, and registered midwives $(3.6 \%, \mathrm{n}=1)$.

Furthermore, most of the midwives $(53.6 \%, \mathrm{n}=15)$ had graduated from college less than five years ago. The number of years in practice as a midwife was less than five for $64.3 \%, \mathrm{n}=18$ of them. The majority $(92.9 \%, \mathrm{n}=$ 26) had only been practicing in their present department or ward for less than five years.

\subsection{Midwives' Awareness of the Components of Standard Preoperative Care for Clients Undergoing EmC/s}

The midwives awareness regarding components of preoperative care is shown in Table 1 . There was sufficient 
Table 1. Midwives' awareness of the components of preoperative care for emergency caesarian section at Bwaila hospital in Malawi.

\begin{tabular}{|c|c|c|c|}
\hline Component & Adherence (\%) & Component & Adherence (\%) \\
\hline Obtain informed consent & 92.9 & Pulse rate check & 25.0 \\
\hline Offer surgery information & 57.1 & Respirations check & 25.0 \\
\hline Allow client to ask questions & 28.6 & Temperature check & 25.0 \\
\hline Offer psychological support & 60.7 & Administer preoperative antibiotics & 82.1 \\
\hline Administer IV fluids & 96.4 & Collect blood specimens & 78.6 \\
\hline Check vital signs & 28.6 & Perform bladder catheterization & 100.0 \\
\hline Bp check & 28.6 & & \\
\hline
\end{tabular}

knowledge among the midwives on only four of the 14 preoperative components (Table 1). The midwives had sufficient knowledge and scored above $80 \%$ in the following preoperative standard of care guidelines; obtaining informed consent $(92.9 \%, \mathrm{n}=13)$, administration of IV fluids $(96.4 \%, \mathrm{n}=13.5)$, administrative of preoperative antibodies $(82.1 \%, \mathrm{n}=11.5)$ and performing bladder catheterization $(100 \%, \mathrm{n}=14)$ (Table 1$)$. The midwives performed below standard on preoperative care guidelines that dealt with the psychological wellbeing and checking of vital signs (Table 1).

\subsection{Preoperative Teaching Offered to Clients}

The midwives scored below $80 \%$ on preoperative teaching that was offered to clients. Only $11.1 \%(n=4)$ knew about teaching their clients on deep breathing and coughing exercises. Teaching on leg exercises was known by only $10.7 \%(n=3)$ of the midwives and only $14.3 \%(n=4)$ of the midwives mentioned frequent turning of clients in bed and early ambulation. Few midwives $(19.2 \%, n=5)$ indicated that they instructed their clients on when to take food or water after surgery.

\subsection{Source of Knowledge on Preoperative Guidelines}

All midwives interviewed $(100 \%, \mathrm{n}=28)$ mentioned that they had not attended any form of in-service training in perioperative care. The source of knowledge for preoperative preparation of clients for $89.3 \%(n=25)$ of the midwives was from pre-service training. The majority $(82.1 \%, n=23)$ did not have preoperative care guidelines in their wards.

\subsection{Midwives Documentation of the Components of Standard Preoperative Care}

The review of clients records showed variation between the results of midwives knowledge from the interviews and actual practice. The midwives failed to document all the component of preoperative care. The highest documented component was informed consent $(77 \%, \mathrm{n}=22)$, followed by IV fluids $(51.7 \%, \mathrm{n}=14)$, bladder catheterisation $(50.6 \%, \mathrm{n}=14)$, and administration of preoperative antibiotics $(37.9 \%, \mathrm{n}=11)$. Documentation was less than $20 \%(n=6)$ for the rest of the components and there was completely no documentation for preoperative teaching on position change, leg exercises, early ambulation, incision area care and when to eat or drink.

\section{Discussion}

\subsection{Demographic Characteristics}

The results of the duration of practice for the midwives in the maternity and antenatal wards show that most of the health workers were less experienced with their work in the maternity wards. The results that most of the care was provided by Nurse Midwife Technicians and Enrolled Nurse midwives have implications on adherence to preoperative standards of care. The Nurse Midwife Technicians and Enrolled Midwives are normally trained to conduct low risk care and deliveries for mothers that did not have any complications when giving birth. On 
the other hand the Registered Midwives are the ones that are trained to assist laboring mothers with pregnancy related complications. Registered midwives have extended and expanded roles and are trained to utilize critical cognitive skills and evidence based knowledge to plan, implement and evaluate maternal and new born care. In addition they are also trained to supervise the enrolled midwife [12]. The number of registered nurses was therefore not adequate for Bwaila maternity unit. Bwaila is a major referral center which receives high risk deliveries, hence needs more registered midwives to cope with the high risk referrals as well as to supervise the lower cadres. Over dependency on novices who had recently qualified to render preoperative care at the facility may explain the substandard performance of the midwives. Similar results were reported from Uganda [13]. One of the recommended strategies to assist novice practitioners to prepare for complex health care is through mentoring partnerships [14]. Therefore there is a need to increase the number of registered midwives so that the novices are paired with more experienced midwives to cope with the demanded standards during EmC/s at the facility.

\subsection{Midwives' Awareness of the Components of Preoperative Care}

The high awareness in some of the components of preoperative care for emergency caesarean section amongst the midwives is commendable. However the inability of most of the midwives to mention that they would allow the client to ask questions and also for them to answer the clients' questions implies that the psychological care of these clients was a neglected part of their preoperative care. These results agree with those reported by Lungu, et al. [14] at the same facility which also shows that clients did not feel comfortable to ask questions and were not given an opportunity to ask questions during antenatal care. The unplanned nature of an emergency caesarean birth means that women do not have a chance to prepare psychologically and hence need some psychological preparation by the midwives. Limited psychological counseling may limit the woman's ability to adjust to the EmC/s.

The results that the midwives performed below standard on the psychological preparation of the clients before $\mathrm{EmC} / \mathrm{s}$ have implications on the clients' perception regarding the quality of care at the facility. There is evidence that many women have tokophobia (anxiety regarding labor and child birth) and that more women undergoing emergency caesarean section express negative feelings towards their delivery as compared to those undergoing elective caesarean section [15]. Some of this anxiety may originate from a previous traumatic birth experience which can overwhelm a woman's normal ability to cope with stress [15].

When this anxiety is manifested in patients undergoing emergency caesarean section it can increase their risks and present added potential for complications both psychological and physiological [16]. In a study done amongst Swedish women by Somera et al. [17] the respondents expressed that asking questions and receiving more information during childbirth would have been more helpful to understand what was going to happen to them. Psychological care of a woman is important for their adaptations. These adaptations occur around the time of delivery and the first weeks of motherhood and are important for the development of the maternal role [18]. Midwives may have performed below standard on psychological preparation of clients because this component is not included in the RH Care Guidelines. There is therefore a need to review the RH guidelines to include this component of preoperative care.

The results that most midwives did not check vital signs on the clients before EmC/s imply that clients at risk for EmC/s were missed out. Generally vital signs provide baseline data which is important for detecting signs of impending deterioration especially in such cases as emergency caesarean section. Knowing the clients' vital signs is important before operation for the detection of overt or covert complications such as hypovolemia, high blood pressure and tachypnoea [18]. The results of this study are in tandem with the Malawi EmONC assessment report [4] which also showed some gaps in the checking of vital signs of clients in the labor wards of most facilities in Malawi. Similar studies have also reported gaps in checking of vital signs amongst nurses in America [19] [20]. There is therefore a need for effective supervision of midwives in the maternity so that all clients are properly checked for vital signs before $\mathrm{EmC} / \mathrm{s}$.

\subsection{Preoperative Teaching Offered to Clients}

Another neglected area on the preoperative guidelines of care was preoperative teaching rendered to the client before EmC/s. Results indicated that this was the most neglected area of care. Furthermore the results show that the type of preoperative teaching that was rendered to clients varied from one midwife to another. These results 
imply that majority of the clients were not properly taught hence they missed out on important information. These results are consistent with those reported by Fitzpatrick and Hyde [21] that a group of nurses in Ireland had varying views on what exactly patient education comprised and had different perceptions on its relative importance as an aspect of preoperative care. Preoperative teaching is beneficial for both patients and the facility in terms of outcomes such as pain, anxiety and rate of recovery [22]. When clients do not get appropriate information from the midwives they tend to get it from the informal support system which creates tension, doubts and anxiety [23]. This component is also not included in the current standard guideline. There is therefore a need to review the current guidelines to include this component. In addition in-service training should to be given to the midwives so that they provide good quality preoperative teaching to clients.

\subsection{Midwives Source of Knowledge for Components of Preoperative Care on Emergency Caesarean Section}

The midwives mostly relied on knowledge from pre-service training during their practice because in-service training sessions on perinatal care were rare. Additionally the results show that the majority of the midwives were not aware of the availability of preoperative care guidelines in their wards. These results agree with those reported by Kaye [13] that lack of in-service training and absence of standard treatment guidelines were major contributing factors for poor quality of care among midwives in Uganda. The fact that few midwives were able to mention the availability of guidelines in their wards suggests that the midwives may not have been properly oriented to the guidelines. It is important that midwives are properly oriented because orientation of midwives and adherence to guidelines is associated with a significant reduction in maternal morbidity [23].

\subsection{Midwives' Documentation of Care Rendered Prior to Emergency Caesarean Section}

The study results showed a wide gap between midwives' awareness of the components of preoperative care and what was actually documented in the clients' charts. A wider gap was noted in components of bladder catheterization where all midwives had shown awareness yet documentation was seen in only half of the charts. Similarly administration of preoperative antibiotics awareness was more than $80 \%$ but documentation was less than $50 \%$. A similar trend was observed in all the other components. It is a well known fact in nursing that what is not documented is considered to have not been done. Results show that though midwives were aware of the components of preoperative care they were not able to put them into practice. These results have adverse consequences on clients' outcomes because despite the debate regarding routine bladder catheterization prior to caesarean section, it has still been proved to be beneficial for the prevention of a full bladder during and immediately after operation [24]. In Malawian hospitals routine bladder catheterization is practiced with all caesarean sections to prevent full bladder that is one of the major causes of $\mathrm{PPH}$, and is one of the leading causes of maternal deaths [25]. For Malawi therefore, the benefits of catheterization outweigh the risks and hence routine bladder catheterization was supposed to be fully documented if it was really done as per the level of awareness among the midwives.

Prophylactic antibiotics administration is important in prevention of post caesarean surgical wound infection, and post caesarean maternal infections such as endometritis, urinary infections, and pneumonia [26] [27]. However, there was low adherence towards this component of the guidelines by some midwives as per the client charts. The results show that the midwives placed the clients that did not receive the antibiotic prophylaxis at high risk of postoperative infections and thus adversely affected the EmC/s outcome. Therefore the preoperative care that was provided to EmC/s clients at Bwaila was below standard and there is a need to train the midwives and intensify supervision of the maternity staff.

\section{Conclusion}

Standard of preoperative care for emergency caesarean section is compromised at Bwaila maternity due to health care provider problems and policy issues. There is need to deploy more Registered Midwives to this hospital to render evidence based preoperative care and supervise the junior cadres who are in large numbers. There is also need to upgrade all the junior midwives who are eligible to Registered Nurse level to ensure delivery of quality evidence based care. The existing preoperative care guidelines need to be revised to include the missing components of preoperative care. There is need for all midwives to be oriented to the guidelines for preoperative 
care and the supervision of midwives to ensure that their knowledge on preoperative care is put into practice.

\section{Study Limitation}

The study was conducted at one facility only and the results may not be applicable to all facilities in Malawi although the trend is similar.

\section{Acknowledgements}

This study was conducted as part of the senior author's Master of Science degree in Midwifery at the University of Malawi, Kamuzu College of Nursing with sponsorship from USAID grant No. REQ-612-12-000036. The preparation of the manuscript for publication was funded by the Norwegian Arctic University at Tromso, Norway and the Agency for Norwegian Development Cooperation under the Maternal and Neonatal Intervention Project in Chiradzulu District.

\section{Conflict of Interest}

The authors have no conflict of interest to disclose.

\section{References}

[1] Van der Putten, D. (2008) The Lived Experience of Newly Qualified Midwives: A Qualitative Study. British Journal of Midwifery, 16, 348-356. http://dx.doi.org/10.12968/bjom.2008.16.6.29592

[2] Berghella, V. (2011) Cesarean Delivery: Preoperative Issues. http://www.uptodate.com/contents/cesarean-delivery-preoperative-issues

[3] Shah, A., Fawole, B., M’Imunya, J.M., Amokrane, F., et al. (2009) Cesarean Delivery Outcomes from the WHO Global Survey on Maternal and Perinatal Health in Africa. International Journal of Gynecology and Obstetrics, 107, 191197. http://dx.doi.org/10.1016/j.ijgo.2009.08.013

[4] Ministry of Health (2010) Malawi 2010 EmONC Needs Assessment final Report. Ministry of Health, Lilongwe.

[5] Fenton, P.M., Whitty, C.J.M. and Reynolds, F. (2003) Caesarian Section in Malawi: Prospective Study of Early Maternal Perinatal Mortality. British Medical Journal, 327, 587. http://dx.doi.org/10.1136/bmj.327.7415.587

[6] American College of Obstetrics and Gynecologists and American Academy of Pediatrics (2007) Guidelines on Perinatal Care. 6th Edition.

[7] Edwards, Z. and McColgan, K (2011) Elective Caesarean Section: A Case Study. Journal of Perioperative Practice, 21, 60-63.

[8] Ministry of Health (2008) Reproductive Health Standards. Ministry of Health, Malawi Ministry of Health, Lilongwe.

[9] Smeltzer, S.C., Bare, B.G. and Hinkle, J.L. (2011) Brunner and Suddarth’s Textbook of Medical-Surgical Nursing. 11th Edition, Lipincott Williams \& Wilkins, Philadelphia.

[10] National Statistical Office (NSO) and ICF Macro (2011) Malawi Demographic and Health Survey 2010. NSO and IFC, Macro, Zomba and Calverton.

[11] Ministry of Health (2005) Malawi National Reproductive Health Service Guidelines. Malawi Ministry of Health, Lilongwe.

[12] Nurses and Midwives Council of Malawi (2008) Scope of Practice for All Cadres of Nurses and Midwives. Nurses and Midwives Council of Malawi, Lilongwe.

[13] Kaye, D. (2008) Quality of Midwifery Care in Soroti District, Uganda. East African Medical Journal, 77, 558-561.

[14] Lungu, F., Malata, A.M. and Chirwa, E. (2011) Quality Assessment of Focused Antenatal Care Services in Malawi. African Journal of Midwifery and Women's Health, 5, 169-174. http://dx.doi.org/10.12968/ajmw.2011.5.4.169

[15] Graham, W.J., Hundley, V. and McChayne, A.L. (2005) An Investigation of Women's Involvement in the Decision to Deliver by Caesarian Section. International Journal of Obstetrics and Gynaecology, 106, 213-220. http://dx.doi.org/10.1111/j.1471-0528.1999.tb08233.x

[16] Pritchard, M. (2009) Managing Anxiety in the Elective Surgical Patient. British Journal of Nursing, 18, 416-419. http://dx.doi.org/10.12968/bjon.2009.18.7.41655

[17] Somera, M.J., Feeley, N. and Ciofani, L. (2009) Women's Experience of an Emergency Caesarean Birth. Journal of Clinical Nursing, 19, 2824-2831. http://dx.doi.org/10.1111/j.1365-2702.2010.03230.x 
[18] Nunney, R. (2008) Providing Perioperative Care for Pregnant Women. Nursing Standard, 22, 40-44. http://dx.doi.org/10.7748/ns2008.07.22.47.40.c6632

[19] Parkes, R (2011) Rate of Respiration, the Forgotten Vital Sign. Emergency Nurse, 19, 12-18. http://dx.doi.org/10.7748/en2011.05.19.2.12.c8504

[20] Smith, S. (2008) An Audit of Nursing Observation on Ward Patients. Nursing Times, 104, 28-29.

[21] Fitzpatrick, E. and Hyde, A. (2005) What Characterizes the "Usual” Preoperative Education in Clinical Contexts. Nursing and Health Sciences, 7, 251-258. http://dx.doi.org/10.1111/j.1442-2018.2005.00244.x

[22] Wiklund, I., Edman, G. and Larsson, C. (2009) First Time Mothers and Changes in Personality in Relation to Mode of Delivery. Journal of Advanced Nursing, 65, 1636-1644.

[23] Bansa, M., Obrien, B. and Oware-Gyekye, F. (2009) Perceived Prenatal Learning Needs of Multigravid Ghanaian Women. Midwifery, 25, 317-326. http://dx.doi.org/10.1016/j.midw.2007.07.006

[24] Nasr, A.M., Elbigawy, A.F. and Abdelamid, A.E. (2009) Evaluation of the Use vs. Nonuse of Urinary Catheterization during Cesarean Delivery: A Prospective, Multicenter, Randomized Controlled Trial. Journal of Perinatology, 29, 416421. http://dx.doi.org/10.1038/jp.2009.4

[25] Lema, V.M., Kanyighe, C. and Changole, J. (2009) HIV/AIDS and Pregnancy Related Deaths in Blantyre, Malawi. East African Medical Journal, 86, 509-512.

[26] Tita, A.T.N., Hauth, J.C. and Grimes, A. (2008) Decreasing Incidence of Post Cesarean Endometritis with Extended-Spectrum Antibiotic Prophylaxis. Obstetrics \& Gynecology, 111, 51-56. http://dx.doi.org/10.1097/01.AOG.0000295868.43851.39

[27] Tita, A.T.N., Rouse, D.J. and Blackwell, S. (2009) Emerging Concepts in Antibiotic Prophylaxis for Cesarean Delivery, A Systematic Review. Obstetrics \& Gynecology, 113, 675-682. http://dx.doi.org/10.1097/AOG.0b013e318197c3b6 
Scientific Research Publishing (SCIRP) is one of the largest Open Access journal publishers. It is currently publishing more than 200 open access, online, peer-reviewed journals covering a wide range of academic disciplines. SCIRP serves the worldwide academic communities and contributes to the progress and application of science with its publication.

Other selected journals from SCIRP are listed as below. Submit your manuscript to us via either submit@scirp.org or Online Submission Portal.
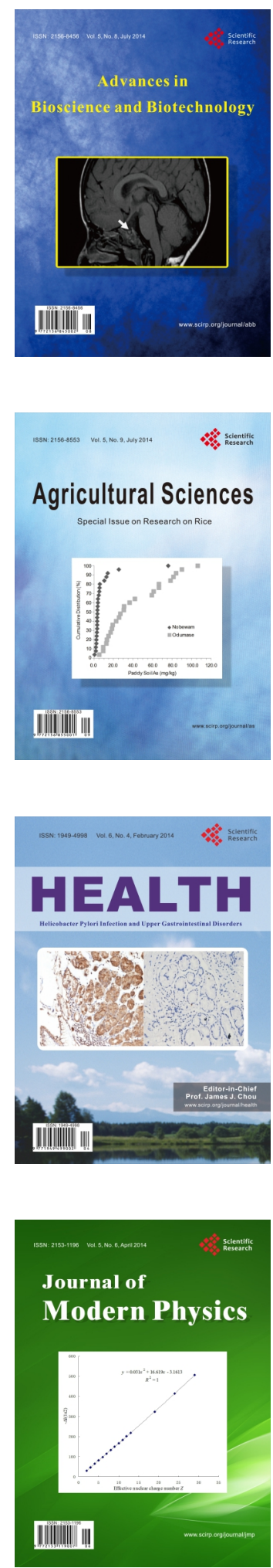
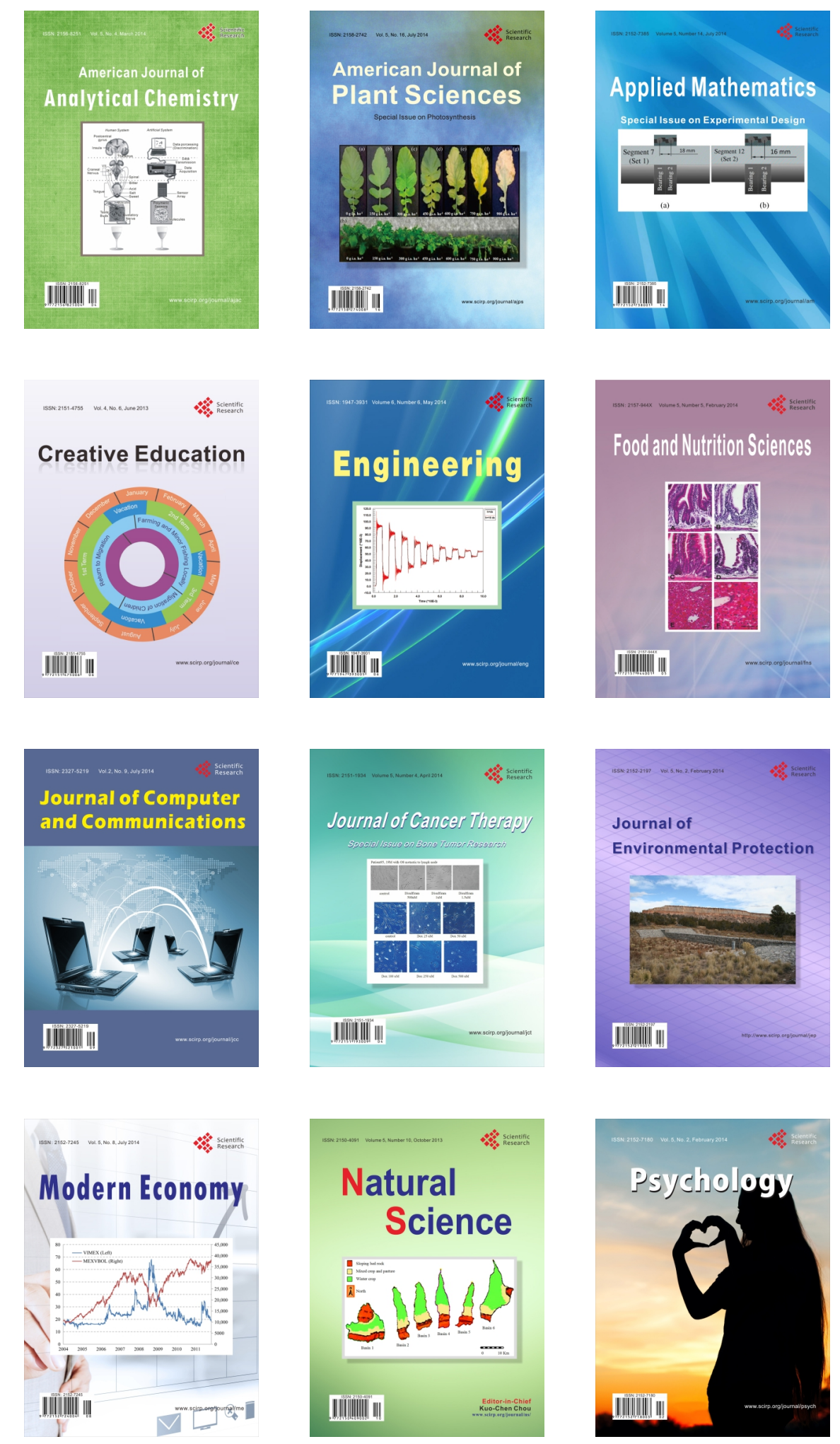